\title{
K-Tools: Towards Semantic Knowledge Management
}

\author{
Sam Chapman ${ }^{1}$, Vitaveska Lanfranchi ${ }^{2}$, and Ravish Bhagdev ${ }^{1,2}$ \\ ${ }^{1}$ Knowledge Now Limited, 217 Portobello, Sheffield, S1 4DP, United Kingdom \\ N. Surname@k-now.co.uk \\ ${ }^{2}$ Department of Computer Science, Regent Court, 211 Portobello, Sheffield, \\ S1 4DP, United Kingdom \\ N.Surname@dcs.shef.ac.uk
}

\begin{abstract}
This paper details the use of Semantic Web tools for supporting networked knowledge acquisition, search and sharing in large distributed organisations. The demonstration will showcase from a user perspective an application developed to aid knowledge management in large organisations, detailing the problems and technical solutions employed.
\end{abstract}

Keywords: Semantic Web, Search, Forms, Knowledge Management, Business Intelligence, K-Now, K-Tools, Knowledge Capture, Enterprise.

\section{Introduction}

The amount of information that large organisations need to collect and store in digital archives and repositories is growing exponentially. Traditional knowledge management and sharing practices fail to address this data explosion: centralised fixed capture methodologies struggle to cope with dynamic, varied and specialised knowledge (i.e. the long tail of an organisations knowledge). Although such knowledge is highly variable it is also essential to specific parts of organisations that want to share and collaborate using it in conjunction with existing centralised knowledge. Many organisations attempt to capture this growing knowledge in shareable digital formats to encourage reuse. Semantic wikis [1] are sometimes utilised but they require training and time to use, lack integration with pre-existing knowledge representations and do not encourage knowledge integration and discovery across an organisation.

We propose a general knowledge lifecycle management methodology where Semantic Web Technologies are adopted to improve efficacy and efficiency in preserving and sharing organisational memory [2]. We therefore introduce a new framework for knowledge management, K-Tools, which uses Semantic Web Technologies to empower distributed networked representation, capturing, retrieval and sharing of knowledge within large enterprises. K-Tools is described in detail (including technologies and implementation choices), followed by the demonstration plan.

\section{K-Tools}

K-Tools is a Semantic Knowledge Management Framework that targets the whole knowledge lifecycle in an easy and usable way, thus maximising the potential adoption inside an organisation. K-Tools is composed of two main technologies: 
1) K-Forms supports knowledge acquisition and representation (the definition of the knowledge structures, e.g. an ontology) as well as capture (the creation of instances);

2) K-Search enables flexible searching and sharing of information and knowledge across differing views, perspectives and documents.

Both tools use the concept of forms as the user interaction paradigm. Form-based solutions are very familiar to users being very common in knowledge management and in every-day life therefore they are very easy to understand and use.

\subsection{Key Technologies Adopted in K-Tools}

K-Forms supports knowledge acquisition allowing easy creation and deployment of web forms that can instantly be used to capture knowledge at its generation time.

Forms are divided into sections and fields. Sections having further subsections and fields, e.g. the section designed to hold data about a person will be a concept; a person will have a series of properties e.g. name, address and date of birth. Sections are presented as sub-forms to be filled. Fields are typed (e.g. text field, integer field, text area, checkbox, option list, etc.). Fields represent meta-properties of the document (e.g. author, date, etc.) or its content (e.g. an issue to be reported). Simple fields will require just the inputting of a value at filling time (a number, a string, a text, a date, etc.). When a field is defined, the user selects a field type and must input appropriate properties for the specific field (e.g. size, validation constraints, default value, help text, etc.). If parts of an existing form are imported, the system automatically imports any associated constraint (e.g. on data types).

When a form is created (see Figure 1), K-Forms translates a template into an OWL Ontology with the form being represented as an OWL class (<FormName $>$ Class) with its own NameSpace to avoid name conflicts. Form sections are represented as OWL classes (<ConceptName> Class) which can have subsections (related classes) or individual fields (properties). Relations between concepts are represented as OWL relations between classes. Adding relations between parents (section or entire form) and the contained subsections (object type properties) enables reuse of entire sections. Relational tables can be represented as advanced sections. The domain of some relations may be the overarching $<$ FormName $>$ Class. When concepts are introduced at the top level, a relation is formally created domain $<$ FormName $>$ Class and range $<$ ConceptName $>$ Class. Individual fields (such as text box, text area, check box) can be added as a property of each section, subsection, or directly in form classes; they are represented as OWL properties. Some of them (as FormCompilationDate) are properties of the overarching <FormName $>$ Class. Restrictions can be set for the possible values of the individual fields using xml datatype schema (for example declaring a type to be a positive integer). Again individual fields can be reused between forms and classes. When concepts are reused across forms, owl:equivalentClass is created. This is due to the fact that uses may reuse the concept but change non conceptual details regarding its visualisation. 


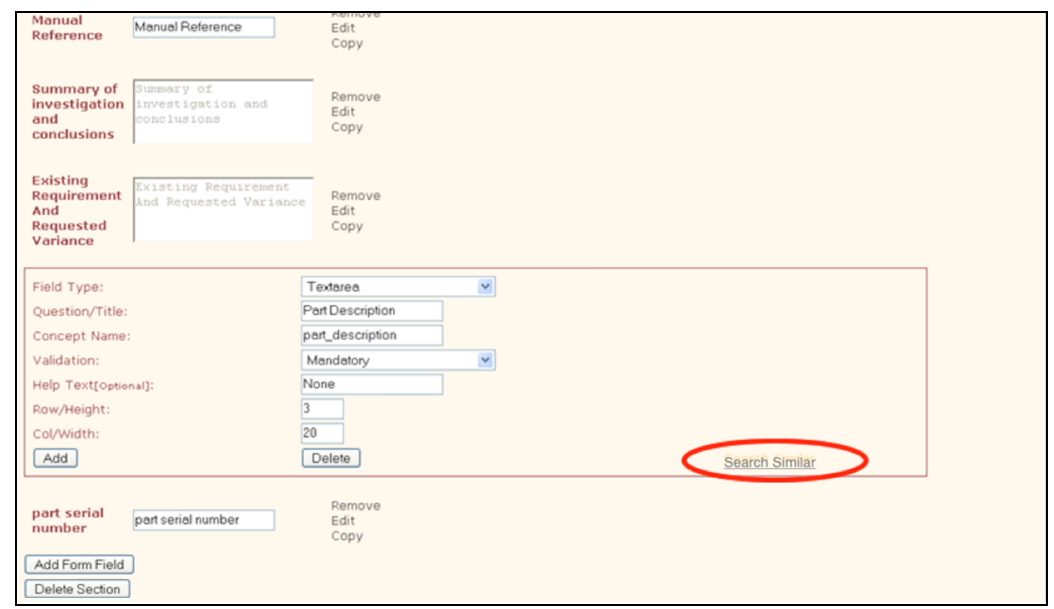

Fig. 1. K-Forms showing the generation of a new form

When (parts of) a form are reused (see Figure 1), an explicit mapping between (parts of) the two underlying ontologies is established. This means that instead of creating new concepts into the form namespace, the new ontology will use the concept in the other form's namespace. If the concept is modified, then a link between the two concepts is maintained by automatically creating a meta-class capturing the common parts. This may happen either at the single concept level or at the whole form level. This creates automatically a network of interconnected ontologies.

Knowledge acquisition using the new form is then realised by transforming all input data into RDF statements related to the form ontology, which can then be searched using standard query languages such as SPARQL and SERQL. This allows all captured knowledge to be automatically linked across an organisation for instantaneous reuse. K-Forms is described in detail in [3].

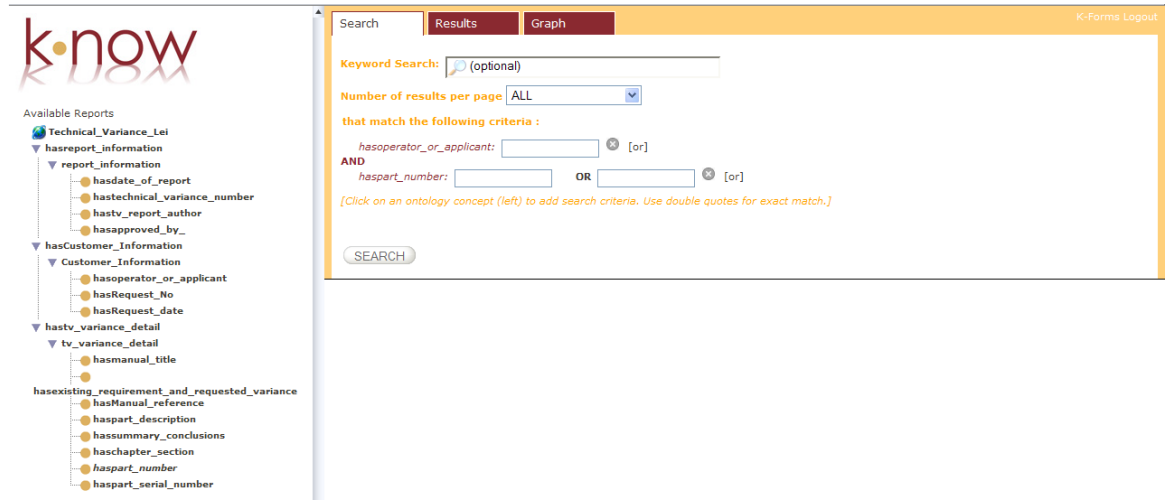

Fig. 2. The Ontology (left) produced by K-Forms after a new form is created can be instantly viewed in K-Search for searching newly generated knowledge 
After knowledge has been stored, it can be searched and explored using K-Search (see Figure 2). Going beyond keyword approaches, K-Search enables Hybrid Search (HS) [4] over multiple repositories at once, from the perspective of a document type, a departmental view, a personal view or from a full company ontology. In HS, users can combine within the same query:

(i) ontology-based search;

(ii) keyword-based search and

(iii) keyword-in-context based search.

Ensuring consistency in the framework, K-Search has been designed following the same form-based interaction paradigm, providing an interface that allows users to flexibly query conditions on both structured knowledge and unbounded keywords within the same query.

The query output of K-Search is a set of ranked documents displayed as a list on the mid-right panel of the interface; each item in the list is identified by the values in the metadata that satisfy the ontology-based search. Moreover, K-Search provides ways of inspecting the results of the query as bi-dimensional graphs (pie or bar chart) according to two elements e.g. issue and component); items in the graph (e.g. pie chart slices) are clickable to focus on the associated subset of data (e.g. associated documents or data). The retrieved triples can be exported if needed (in RDF, CSV or XLS formats) for external analysis or automated processing. K-Search is described in details in [4].

\section{System Demonstration}

The demonstration focuses on showing how K-Tools fit the dynamic needs of large organisations, collecting knowledge across multiple sites/departments with various changing focuses. For demonstration purposes, K-Tools will utilise data from preexisting industrial applications and then show in real-time how pre-existing forms can be modified or reused and how newly created content can be queried in a flexible way alongside pre-existing knowledge.

The system will be demonstrated from a user perspective, showing through $\mathrm{K}$ Tools interfaces how a single user within a large organisation can create forms, distribute them, fill them and search the available knowledge. The underlying technology will be explained at each stage (although normally hidden in such a way as to allow any user within an organisation to use the system with minimal training).

Firstly from the point of capturing new knowledge relevant to an individuals task, e.g. a simple form design process whereby a user can create/reuse/modify fields or sections of forms previously entered and then use them as they wish. This wide flexibility helps addressing the long tail of organisational knowledge allowing large companies to capture specific knowledge across departments/groups/users. It will be shown how a user can easily create, modify and reuse form components by selecting some simple properties (e.g. type, name, validation constraints, default value, help text, etc.) - the underlying ontology(-es) will then be shown to better showcase the semantic mapping processes happening in background. 
For the knowledge capturing stage, during the demonstration it will be possible to fill in created forms and then analyse the RDF triples that have been automatically generated by the system.

Knowledge sharing and reuse will be demonstrated through K-Search, showing how the previously created/modified forms and the collected information are immediately available for exploration by keyword, semantic and hybrid queries in a single interface. Most importantly this demonstration will highlight real world cases where semantics alone cannot solve a problem and show how integration with legacy data/systems aids in organisational adoption. The hybrid search modality will be showcased to explain how K-Search bridges the gaps with pre-existing unstructured knowledge and how it is possible to search information that is not fully represented by the available ontologies.

Acknowledgments. The work was supported by 1) IPAS, a project jointly funded by the UK DTI (Ref. TP/2/IC/6/I/10292) and Rolls-Royce plc, 2) X-Media, an Integrated Project on large scale knowledge management across media, funded by the European Commission as part of the IST programme (IST-FP6-026978), (www.x-mediaproject.org) and 3) Knowledge Now Ltd.

\section{References}

1. Krötzsch, M., Vrandecic, D., Völkel, M., Haller, H., Studer, R.: Semantic Wikipedia. Journal of Web Semantics 5, 251-261 (2007)

2. Stern, E.W.: Organizational memory: Review of concepts and recommendations for management. International Journal of Information Management, 17-32 (1995)

3. Bhagdev, R., Chakravarthy, A., Chapman, S., Ciravegna, F., Lanfranchi, V.: Creating and Using Organisational Semantic Webs in Large Networked Organisations. In: Sheth, A., et al. (eds.) ISWC 2008. LNCS, vol. 5318, pp. 723-736. Springer, Heidelberg (2008)

4. Bhagdev, R., Chapman, S., Ciravegna, F., Lanfranchi, V., Petrelli, D.: Hybrid Search: Effectively Combining Keywords and Semantic Searches. In: Bechhofer, S., Hauswirth, M., Hoffmann, J., Koubarakis, M. (eds.) ESWC 2008. LNCS, vol. 5021, pp. 554-568. Springer, Heidelberg (2008) 